\title{
Case Report \\ Successfully treated spondylodiscitis due to a previously unreported mycobacterium
}

\author{
Enrico Tortoli, ${ }^{1,2}$ Antonia Mantella, ${ }^{3}$ Alessandro Mariottini, ${ }^{1,4}$ \\ Gianna Mazzarelli, ${ }^{1,5}$ Patrizia Pecile, ${ }^{2}$ Pier G. Rogasi, ${ }^{6}$ Gaetana Sterrantino, ${ }^{6}$ \\ Elisa Fantoni ${ }^{6}$ and Francesco Leoncini ${ }^{6}$ \\ 1,2,4,5,6 Regional Reference Centre for Mycobacteria ${ }^{1}$, Microbiology and Virology Laboratory ${ }^{2}$, \\ Cytogenetics and Genetics Unit4, Microbiological and Virological Serum-Immunology \\ Laboratory ${ }^{5}$ and Infectious Diseases Unit6, Careggi Hospital, viale Morgagni 85, \\ 50134 Florence, Italy \\ ${ }^{3}$ Infectious Diseases Unit, University of Florence, Florence, Italy
}

Correspondence

Enrico Tortoli

e.tortoli@libero.it

Received 20 July 2005

Accepted 31 August 2005
A non-tuberculous mycobacterium was isolated, following a vertebral needle aspiration, from the blood of a patient with severe spondylodiscitis. The strain turned out to be different from any known mycobacterial species and was quite drug-susceptible in vitro. The patient improved markedly following treatment with meropenem, clarithromycin and amikacin.

\section{Introduction}

The rapidly growing non-tuberculous mycobacteria were considered for many years to be minor members of the genus Mycobacterium, with only a few species recognized, and those often incorrectly grouped together in the Mycobacterium fortuitum-Mycobacterium chelonae complex (Wolinsky, 1979; Wayne \& Sramek, 1992). In recent years, however, a quite different scenario has emerged mainly thanks to the investigations of Wallace and co-workers (Brown-Elliott \& Wallace, 2002). Not only has the number of rapidly growing species identified increased considerably, but the prevalence of clinically significant infections due to such organisms has also become clear. The present case joins the large number of mycobacterial pathologies involving joints and bone (Brown-Elliott \& Wallace, 2002).

\section{Case report}

A 61 year old male suffering from cirrhosis was hospitalized because of severe backache. Radiographic and tomographic pictures revealed clear signs of infection, with massive destruction of the 3 rd and 4 th dorsal vertebrae (Fig. 1). A needle biopsy yielded purulent material which was cultured for common bacteria without isolation of significant pathogens (rare colonies of coagulase-negative staphylococcus). Culture for mycobacteria was not done. The day after the intervention, because of a sudden rise in the patient's temperature, three blood cultures were made for aerobic and anaerobic bacteria. An empirical treatment with

Abbreviation: ITS, internal transcribed spacer.

The GenBank/EMBL/DDBJ accession numbers for the partial $16 S$ rRNA gene and 16S-23S ITS sequences of strain Fl-05038 are DQ067465 and DQ185131. meropenem ( $1 \mathrm{~g}$ i.v. $\mathrm{q} 8 \mathrm{~h}$ ), undertaken at the same time, turned out to be effective, with rapid disappearance of the fever. The isolation of an unidentified mycobacterium from all three blood cultures for aerobic bacteria led to the addition to the treatment of clarithromycin $(500 \mathrm{mg}$ i.v. bid) and amikacin ( $1 \mathrm{~g}$ i.v. die), to which the strain was susceptible in vitro. The patient was discharged after 56 days with prescription of protracting the treatment for 4 months. At a 15-week check-up, the patient did not report any fever relapse, while the backache had improved substantially.

\section{Microbiological investigation}

In the case described here, a previously unreported rapidly growing mycobacterium was responsible for spondylodiscitis in an elderly man. Although neither the biopsy specimen

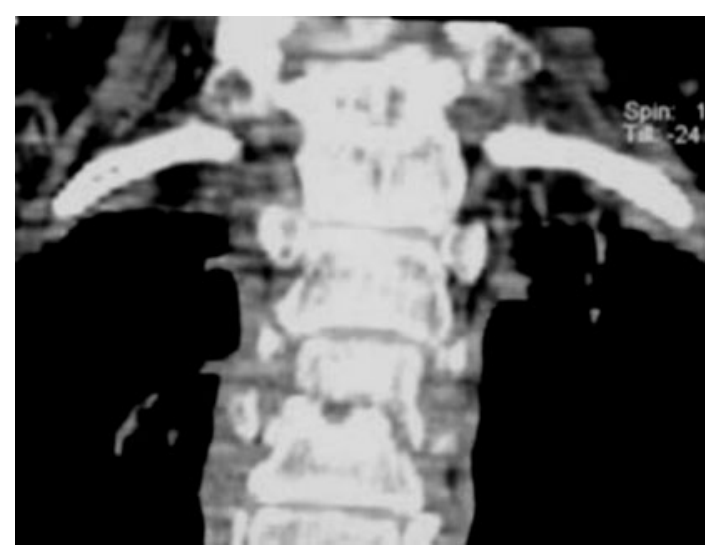

Fig. 1. Tomographic scan revealing massive destruction of vertebral bodies. 


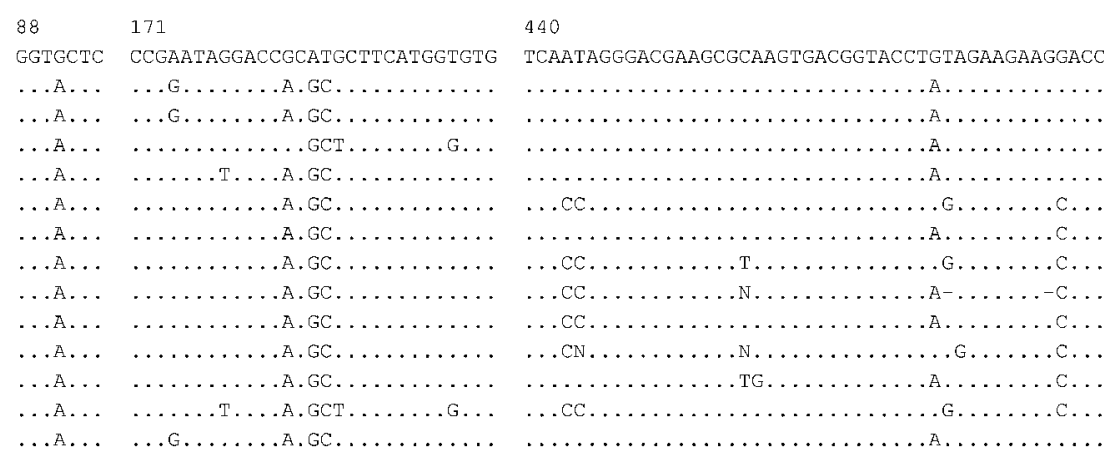

Fig. 2. Alignment of regions of the $16 \mathrm{~S}$ rRNA gene sequence able to differentiate strain $\mathrm{Fl}-05038$ from $M$. farcinogenes, $M$. fortuitum (three different sequevars), M. mucogenicum (all sequevars available in GenBank) and M. senegalense. Base pair positions are indicated according to the Escherichia coli sequence. a, Sequences taken from Hall et al. (2003); $b$, from unpublished GenBank accession number AY627003.

nor the blood of the patient were cultured for mycobacteria, the infection could be diagnosed thanks to the ability of the strain to grow in three blood-culture bottles specific for aerobic organisms (Bactec Plus Anaerobic/F; Becton Dickinson) taken at $30 \mathrm{~min}$ intervals when the patient was feverish. All bottles were scored positive by the automatic instrumentation Bactec 9240 (Becton Dickinson) within 2 to 4 days of incubation, and smooth, white colonies, which turned out to belong to a Gram-uncertain rod (strain FI-05038), grew on a blood agar subculture in 2 days at $37^{\circ} \mathrm{C}$. Because of the failure to obtain a confident identification with conventional phenotypic methods, the sequence of the first third of the 16S rRNA gene of the organism was determined (Cloud et al., 2002). On the basis of this sequence (GenBank accession no. DQ067465), the strain was shown to belong to the genus Mycobacterium but differed from any known species. The most closely related taxa were Mycobacterium farcinogenes, M. fortuitum, Mycobacterium mucogenicum, Mycobacterium porcinum and Mycobacterium senegalense, all differing by 6 bp in a 466 bp region of the 16S rRNA gene sequence (Fig. 2). The partial sequence of the 16S-23S internal transcribed spacer (ITS) (GenBank accession no. DQ185131) was also different from that of any other organism, with the closest one (M. mucogenicum) differing by $34 \mathrm{bp}$ out of $360 \mathrm{bp}$ investigated.

A Ziehl-Neelsen smear confirmed that the organism was acid-fast. Phenotypic investigations revealed that the strain was non-photochromogenic and produced smooth colonies in 2 days at $25-37^{\circ} \mathrm{C}$; it was furthermore able to grow on MacConkey agar without crystal violet but was inhibited by cephalothin (Wallace et al., 1993) and on LowensteinJensen agar with $5 \% \mathrm{NaCl}$. Among biochemical tests, Tween 80 hydrolysis, 3-day arylsulfatase and urease were positive, while nitrate reduction was negative. Catalase activity was low (less than $45 \mathrm{~mm}$ foam). HPLC analysis revealed a pattern of cell-wall mycolic acids roughly compatible with that of M. mucogenicum but clearly different from those of the other species most closely related at the genetic level (Fig. 3).
Susceptibility testing performed using the agar elution method (Brown et al., 1992) revealed effectiveness of amikacin $\left(\leqslant 6 \mu \mathrm{g} \mathrm{ml}^{-1}\right)$, cefoxitin $\left(\leqslant 30 \mu \mathrm{g} \mathrm{ml}^{-1}\right)$, ciprofloxacin $\left(\leqslant 2 \mu \mathrm{g} \mathrm{ml}^{-1}\right)$, clarithromycin $\left(\leqslant 2 \mu \mathrm{g} \mathrm{ml}^{-1}\right)$, doxycycline $\left(\leqslant 6 \mu \mathrm{g} \mathrm{ml}^{-1}\right)$, imipenem $\left(\leqslant 8 \mu \mathrm{g} \mathrm{ml}^{-1}\right)$ and tobramycin $\left(\leqslant 8 \mu \mathrm{g} \mathrm{ml}^{-1}\right)$, with the strain being resistant to trimethoprim/sulfamethoxazole $\left(\geqslant 30 \mu \mathrm{g} \mathrm{ml}^{-1}\right)$ only. Imipenem is usually the only carbapenem tested against rapidly growing mycobacteria; at clinicians' request, we also added meropenem and ertapenem, and both turned out to be active $\left(\leqslant 8 \mu \mathrm{g} \mathrm{ml}^{-1}\right)$.

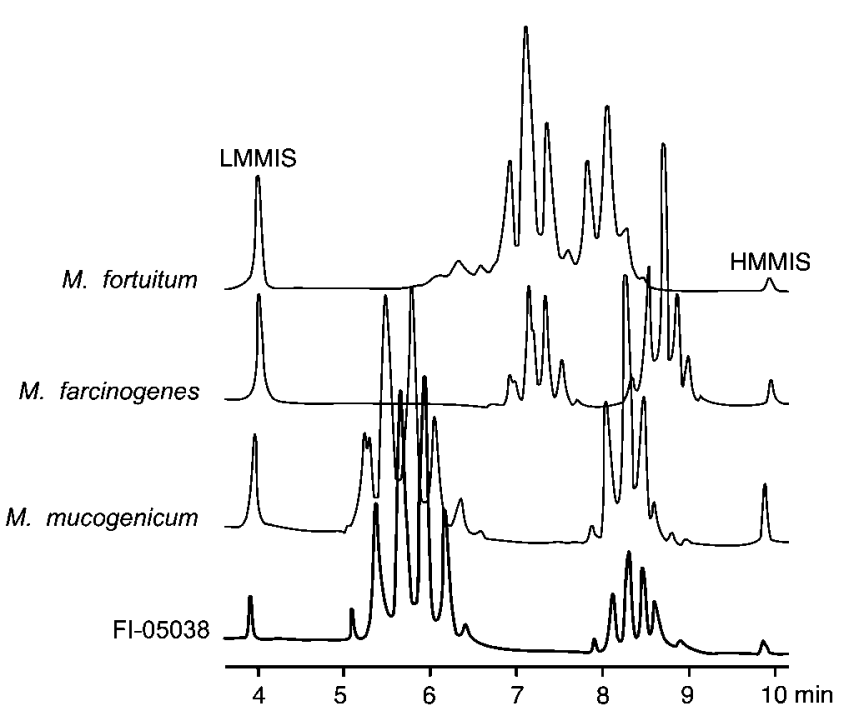

Fig. 3. Representative HPLC pattern of cell-wall mycolic acids of strain $\mathrm{Fl}-05038$ in comparison with those of M. mucogenicum, M. fortuitum and M. farcinogenes. LMMIS, Low-molecular-mass internal standard; HMMIS, high-molecular-mass internal standard. The patterns of $M$. porcinum and $M$. senegalense have not been reported as overlapping substantially with that of $M$. fortuitum. 


\section{Discussion}

We think, in this case, that the presence of the strain in the blood was accidental, having been very likely released from the vertebral lesion as a consequence of the trauma of the needle aspiration. The present isolation, obtained from a sterile site, unquestionably fulfils the American Thoracic Society criteria for clinical significance (American Thoracic Society, 1997).

The growth of several mycobacteria in non-dedicated bloodculture bottles (Jacomo et al., 1998) or on blood agar plates (Drancourt et al., 2003) has already been reported. In this case, this less known feature, along with a number of lucky coincidences, allowed a severe bone infection to be diagnosed and cured. Once again, the too-often disregarded usefulness of extending the microbiological investigation of biopsy samples to mycobacteria has been confirmed.

Once we had excluded the possibility that the test strain belonged to the species $M$. farcinogenes, M. fortuitum, $M$. porcinum and $M$. senegalense on the basis of the phenotypic and genotypic differences, the possibility remains that it is a previously unreported variant of $M$. mucogenicum. The agreement of most phenotypic features (Springer et al., 1995; Wallace et al., 1993) supports such a hypothesis. On the other hand, $1 \cdot 3$ and $10 \cdot 1 \%$ mismatches in the investigated regions of the 16S rRNA gene and 16S-23S ITS, respectively, and the conflicting results of cephalothin tests (susceptibility to this antibiotic is very consistent in $M$. mucogenicum; Springer et al., 1995) favour the suggestion that it belongs to an as-yet unrecognized species. We feel that our current knowledge is not sufficient to make such a decision; furthermore, according to our convictions (Tortoli, 2003), the description of a novel species should not be based on a single strain. At the same time, we are confident that this report will stimulate others to characterize similar strains, increasing our knowledge of this organism.

The case reported here supports a large literature base (Brown-Elliott \& Wallace, 2002) that contradicts the widespread conviction that rapidly growing non-tuberculous mycobacteria almost always play the role of a contaminant in clinical specimens.

\section{References}

American Thoracic Society (1997). Diagnosis and treatment of disease caused by nontuberculous mycobacteria. Am J Respir Crit Care Med 156, S1-S25.

Brown, B. A., Swenson, J. M. \& Wallace, R. J., Jr (1992). Agar disk elution test for rapidly growing mycobacteria. In Clinical Microbiology Procedure Handbook, pp. 5.10.1-5.10.11. Edited by H. D. Isenberg. Washington, DC: American Society for Microbiology.

Brown-Elliott, B. A. \& Wallace, R. J., Jr (2002). Clinical and taxonomic status of pathogenic nonpigmented or late-pigmenting rapidly growing mycobacteria. Clin Microbiol Rev 15, 716-746.

Cloud, J. L., Neal, H., Rosenberry, R., Turenne, C. Y., Jama, M., Hillyard, D. R. \& Carroll, K. C. (2002). Identification of Mycobacterium spp. by using a commercial $16 \mathrm{~S}$ ribosomal DNA sequencing kit and additional sequencing libraries. J Clin Microbiol 40, 400-406.

Drancourt, M., Carrieri, P., Gevaudan, M. J. \& Raoult, D. (2003). Blood agar and Mycobacterium tuberculosis: the end of a dogma. J Clin Microbiol 41, 1710-1711.

Hall, L., Doerr, K. A., Wohlfiel, S. L. \& Roberts, G. D. (2003). Evaluation of the MicroSeq system for identification of mycobacteria by $16 \mathrm{~S}$ ribosomal DNA sequencing and its integration into a routine clinical mycobacteriology laboratory. J Clin Microbiol 41, 1447-1453.

Jacomo, V., Musso, D., Gevaudan, M. J. \& Drancourt, M. (1998). Isolation of blood-borne Mycobacterium avium by using the nonradioactive BACTEC $9000 \mathrm{MB}$ system and comparison with a solid-culture system. J Clin Microbiol 36, 3703-3706.

Springer, B., Böttger, E. C., Kirschner, P. \& Wallace, R. J., Jr (1995). Phylogeny of the Mycobacterium chelonae-like organism based on partial sequencing of the $16 \mathrm{~S}$ rRNA gene and proposal of Mycobacterium mucogenicum sp. nov. Int J Syst Bacteriol 45, 262-267.

Tortoli, E. (2003). Impact of genotypic studies on mycobacterial taxonomy: the new mycobacteria of the 1990s. Clin Microbiol Rev 16, 319-354.

Wallace, R. J., Jr, Silcox, V. A., Tsukamura, M., Brown, B. A., Kilburn, J. O., Butler, W. R. \& Onyi, G. (1993). Clinical significance, biochemical features, and susceptibility patterns of sporadic isolates of the Mycobacterium chelonae-like organism. J Clin Microbiol 31, 3231-3239.

Wayne, L. G. \& Sramek, H. A. (1992). Agents of newly recognized or infrequently encountered mycobacterial diseases. Clin Microbiol Rev 5, 1-25.

Wolinsky, E. (1979). Nontuberculous mycobacteria and associated diseases. Am Rev Respir Dis 119, 107-159. 